CERN-TH/2000-061

hep-th/0002198

\title{
On Type II NS5-branes in the presence of an RR field
}

\author{
Mohsen Alishahiha \\ Theory Division, CERN \\ CH-1211, Geneva, 23, Switzerland
}

\begin{abstract}
In this letter we use Type II NS5-branes in the presence of an RR field at the decoupling limit to study a non-commutative version of little string theory. We shall see that the decoupling limit of NS5-branes in the presence of an RR field is completely different from the one we had in ordinary little string theory; in particular for Type IIA NS5-branes this decoupling limit can be defined only when the theory is wrapped on a circle, but nevertheless flows to the ordinary non-compact $(0,2)$ theory at the IR limit. We also see that these theories, in the UV regime, where the non-commutative effects are important, can be described by smeared ordinary D3(D2)-branes in Type IIB(A) string theory.
\end{abstract}

February 2000 


\section{Introduction}

It was shown in [1] ( see also [2]) that, when we turn on a B field on the D-brane worldvolume, the low-energy effective worldvolume theory is modified to be a noncommutative Super-Yang-Mills (NCSYM) theory. In fact the worldvolume theory of $N$ coincident $\mathrm{D}_{p}$-branes in the presence of a B field is found to be $U(N)$ NCSYM theory [3].

As is the case with a zero B field, there exists a limit where the bulk modes decouple from the worldvolume non-commutative field theory [3, 4]; we expect to have a correspondence between string theory on the curved background with B field and non-commutative field theories. In other words we expect to have a holographic picture like AdS/CFT correspondence [5] (for review and complete list of references, see [6]) for the non-commutative theories. In fact this issue has been investigated in [7]-[12].

It was also shown that there is a limit in which the theory living on the NS5branes decouples from the bulk [13]| It is believed that the decoupled theory is a string theory without gravity called "Little String Theory" (LST) \&. Upon compactification this theory inherits the T-duality of Type II string theories and must therefore be a non-local theory. The simplest way to find this decoupling limit is to start with the decoupling of D5-branes and using S-duality. The decoupling limit of D5-branes is defined by $l_{s} \rightarrow 0$ keeping $g_{Y M}^{2}=g_{s} l_{s}^{2}$ fixed. Using S-duality P one finds

$$
g_{s}^{\prime} \rightarrow 0 \quad g_{Y M}^{2}=l_{s}^{\prime 2}=\text { fixed }
$$

which is the decoupling limit of NS5-branes [13]. Using T-duality we can also obtain the limit in which the Type IIA NS5-branes decouple from the bulk, which is the same as the IIB one (11).

The NS5-branes theory, or LST, has been studied from several points of view; in particular it has been considered in [17] using the holographic principle for string theory in an asymptotic linear dilaton background. There, the authors considered the string theory in the near-horizon background of parallel NS5-branes, which is dual to LST. This duality could help them to study some observables of the theory as well as some of their correlation functions. The theory on $N$ IIA NS5-branes has also been studied at large $N$ using supergravity in [18].

It is a natural question to ask what the non-commutative deformation of LST is. In the spirit of what we have learnt about D-branes in the presence of a B field and its relation with non-commutative gauge theory due to holography (AdS/CFT correspondence), we would expect that the non-commutative deformation of LST could be obtained by NS5-branes in the presence of a non-zero RR field along its

\footnotetext{
1 This idea, that the theory on the NS5-branes could be some sort of string theory, has been also considered in 114, 15] in the context of Matrix theory description of M-theory and its compactifications.

${ }^{2}$ For a brief review, see 16 .

${ }^{3}$ Under S-duality we have $l_{s}^{2} \rightarrow l_{s}^{2} \equiv g_{s} l_{s}^{2}$ and $g_{s} \rightarrow g_{s}^{\prime} \equiv \frac{1}{g_{s}}$
} 
worldvolume. In fact such a background has been considered in [10], and it is the aim of this letter to study this theory in more detail.

The non-commutative deformation of LST is also expected from the DLCQ description of LST [19]; there the authors showed that blowing up the singularities by FI terms can give, in the DLCQ context, a space-time interpretation that could be considered as turning on an RR field parallel to the NS5-branes. Therefore we would expect to find a non-commutative version of LST (NLST).

In this letter we study the theory living on the NS5-branes in the presence of an $\mathrm{RR}$ field at its decoupling limit. We shall only consider the case with the smallest rank of non-zero RR field. This theory can be considered as a non-commutative deformation of little string theory, although as we shall see the decoupling limits of non-commutative and ordinary LST are completely different. In particular in this case the decoupling limit is defined as a limit where the string scale goes to zero. Although even in this limit we have a scale in the theory (coming from the RR field), it is not clear whether the theory on the "non-commutative NS5-branes" obtained in this way is a string theory. In fact, as we shall see, the theory seems to be equivalent to the SYM theory. This may be because of our definition of the decoupling limit of the theory, but till now, it is the only consistent way this decoupling limit can be defined. Nevertheless this theory has some properties of LST; for example we would expect that the thermodynamical quantities of both theories be the same, both theories are non-local and have some sort of T-duality. It might also be possible that what we are studying here is not really a non-commutative deformation of LST. In other words, what we have obtained by S- or T-duality as a decoupling limit of the NS5-branes would be a limit that leads to a non-commutative field theory, such as SYM theory on the NS5-branes with an RR field, and in fact it might be that the non-commutative little string theory does not have a supergravity descriptionf.

In section 2 we shall consider the Type IIB NS5-branes in the presence of an RR field and we will see that in the UV limit where non-commutative effects are important the theory can be described by smeared D3-branes. In section 3 we will study Type IIA NS5-branes. We find that the decoupling limit of the theory is consistent only when the theory is wrapped on a circle and this theory can be described at UV by smeared D2-branes. We will give a conclusion and some comments in section 4 .

\section{Non-commutative Type IIB NS5-branes}

\section{Decoupling limit}

As for the ordinary NS5-brane, where there was a non-trivial theory on its worldvolume in its decoupling limit, we would expect to find a non-commutative version of little string theory on the worldvolume of the NS5-brane in the presence of a non-zero RR field in its decoupling limit. The simplest way to define this theory is to start with a D5-brane in Type IIB string theory in the presence of a non-zero

\footnotetext{
${ }^{4}$ This point was suggested by Y. Oz.
} 
B field along its worldvolume and using S-duality to map the theory to its S-dual. Doing so we end up with the NS5-brane solution with an RR field background.

The decoupling limit of D5-branes in the presence of a large $\mathrm{B}$ field is defined as a limit where $l_{s} \rightarrow 0$ while $g_{s}$ and $b=l_{s}^{2} B$ are kept fixed; moreover, we have to rescale the directions in which the $\mathrm{B}$ field is defined, thereby making them noncommutative. In this limit the modes on the D5-brane decouple from modes on the bulk and we are left with 6-dimensional non-commutative SYM with gauge coupling $g_{Y M}^{2} \sim g_{s} b$. The phase diagram of this theory has been studied in [10].

Using S-duality we can find a decoupling limit in which we expect to have a decoupled theory on the worldvolume of the NS5-brane in the presence of an RR field:

$$
l_{s}^{\prime} \rightarrow 0, \quad b^{\prime}=g_{s}^{\prime} l_{s}^{\prime 2} A=\text { fixed, } \quad g_{s}^{\prime}=\text { fixed }
$$

where $A$ is the RR field obtained from a $\mathrm{B}$ field by $\mathrm{S}$-duality. The coupling of the theory will be $g_{Y M}^{2}=\frac{b}{g_{s}^{\prime}}=b^{\prime}$.

We note that the decoupling limit of the NS5-brane with the RR field is completely different from ordinary NS5-brane (11). In fact, in both the ordinary case and the case with an RR field, what we want to send to zero is $g_{s}^{\prime} l_{s}^{2}$, but the important point is which quantity we would like to keep fixed. Since we want to have a nontrivial theory at the decoupling limit, it is natural to assume that the coupling of the theory should be fixed. In general for an RR field with rank $2 m$, the coupling is proportional to $g_{s}^{-m} l_{s}^{2-2 m}$. Now we can see that adding a non-zero RR field will change the decoupling limit in a completely non-trivial way.

We also note that the decoupling limit of NLST looks very much like ordinary D3-branes. We will go back to this point later.

\section{Supergravity description}

We can study $N$ coincident NS5-branes in the presence of an RR field at large $N$ using supergravity. In order to study this theory one can start with the supergravity solution of D5-branes with a B field and using S-duality. The supergravity solution of D5-branes in the presence of a rank-two $\mathrm{B}$ field is given by:

$$
\begin{aligned}
d s^{2} & =f^{-1 / 2}\left[-d x_{0}^{2}+\cdots+h\left(d x_{4}^{2}+d x_{5}^{2}\right)\right]+f^{1 / 2}\left(d r^{2}+r^{2} d \Omega_{3}^{2}\right), \\
f & =1+\frac{N g_{s} l^{2}}{\cos \theta r^{2}}, \quad h^{-1}=\sin ^{2} \theta f^{-1}+\cos ^{2} \theta \\
B_{45} & =\frac{\sin \theta}{\cos \theta} f^{-1} h, \quad e^{2 \phi}=g_{s}^{2} f^{-1} h .
\end{aligned}
$$

Under S-duality we have

$$
e^{\phi} \rightarrow e^{\phi^{\prime}} \equiv e^{-\phi}, \quad d s^{2} \rightarrow d s^{2} \equiv g_{s} e^{-\phi} d s^{2} .
$$

Using (4) we get the Type IIB NS5-branes background [10]:

$$
d s^{\prime 2}=h^{-1 / 2}\left[-d x_{0}^{2}+\cdots+h\left(d x_{4}^{2}+d x_{5}^{2}\right)+f\left(d r^{2}+r^{2} d \Omega_{3}^{2}\right)\right],
$$




$$
\begin{aligned}
f & =1+\frac{N l_{s}^{2}}{\cos \theta r^{2}}, \quad h^{-1}=\sin ^{2} \theta f^{-1}+\cos ^{2} \theta, \\
e^{2 \phi^{\prime}} & =g_{s}^{\prime 2} f h^{-1},
\end{aligned}
$$

and the NS field $B_{i j}$ is mapped to the RR field $A_{i j}$. The decoupling limit is derived by applying S-duality on the decoupling limit of the D5-branes. As we said above, it is defined by taking the limit ${l^{\prime}}_{s}^{2} \rightarrow 0$ and keeping fixed

$$
\begin{array}{ll}
u=\frac{r}{l_{s}^{\prime 2}} & \bar{g}_{s}^{\prime}=g_{s}^{\prime-1} \\
b^{\prime}=l_{s}^{\prime 2} \tan \theta & \bar{x}_{4,5}=\frac{b^{\prime}}{l_{s}^{\prime 2}} x_{4,5} .
\end{array}
$$

Keeping $u$ fixed means keeping fixed the mass of a D-string stretched between two NS5-branes. In this limit the supergravity solution reads

$$
\begin{aligned}
d s^{\prime 2} & =\frac{l^{\prime 2}}{b^{\prime}} h^{1 / 2}\left[-d x_{0}^{2}+\cdots+h^{-1}\left(d x_{4}^{2}+d x_{5}^{2}\right)+\frac{N b^{\prime}}{u^{2}}\left(d u^{2}+u^{2} d \Omega_{3}^{2}\right)\right], \\
h & =1+(a u)^{2}, \quad a^{2}=\frac{b^{\prime}}{N}, \quad e^{2 \phi^{\prime}}=g_{s}^{\prime 2} \frac{1+(a u)^{2}}{(a u)^{2}} .
\end{aligned}
$$

The curvature of the metric reads

$$
l_{s}^{\prime 2} \mathcal{R} \sim \frac{1}{N} \frac{1}{\left(1+(a u)^{2}\right)^{1 / 2}} .
$$

When $a u \gg 1$, which we are interested in, the supergravity approximation can be trusted for finite $N$, which means that we can study NLST even for finite $N$. In this limit the background reads

$$
\begin{aligned}
l_{s}^{\prime 2} d s^{\prime 2} & =\frac{u}{R^{2}}\left(-d x_{0}^{2}+\cdots+d x_{3}^{2}\right)+\frac{R^{2}}{u}\left(d u^{2}+u^{2} d \Omega_{3}^{2}\right)+\frac{R^{2}}{b^{\prime 2} u}\left(d x_{4}^{2}+d x_{5}^{2}\right) \\
e^{2 \phi^{\prime}} & =\bar{g}_{s}^{\prime 2}, \quad R^{2}=\sqrt{N b^{\prime}},
\end{aligned}
$$

and the curvature of the metric is $l_{s}^{\prime 2} \mathcal{R} \sim \frac{1}{R^{2} u}$. From the dilaton we can see that this solution is similar to D3-branes and it is in fact the D3-branes solution smeared in two directions and without RR field.

The same situation has also been studied for $\mathrm{D}_{p}$-branes in [20, 21]. There, it was shown that the non-commutative $(p+1)$-dimensional SYM theory can be considered as $(p-1)$-dimensional ordinary YM theory whose gauge group is obtained by the $\mathrm{B}$ field. In fact what the authors have shown is as follows: they observed that for $\mathrm{D}_{p^{-}}$ branes in the presence of a B field with rank two at UV, where the non-commutative effects are important, the supergravity solution reduces to $\mathrm{D}_{(p-2)}$-branes smeared in two directions without $\mathrm{B}$ field. In fact this was the case because in this limit the physics is described by $\mathrm{D}_{(p-2)}$-branes; since in this case the $\mathrm{B}$ field that we started 
with is not along the world-volume of the $\mathrm{D}_{(p-2)}$-branes, it can be gauged away, and we end up with an ordinary smeared brane.

In our example the situation is the same as D5-branes where the theory can be described by smeared D3-branes, but in our case we have to gauge away the RR field instead of the B field, which is made possible by the $S L(2, Z)$ symmetry of Type IIB string theory. We also note that the smeared D3-brane is also self-dual under Sduality. The reason is that, in smeared D3-branes, only the harmonic function of the metric will change and the dilaton and 4-form field will be the same as localized D3branes. Therefore we would expect that the $S L(2, Z)$ symmetry maps the smeared D3-branes to itself.

Since both the non-commutative D5-branes theory and the non-commutative NS5-branes can be described by ordinary D3-branes smeared in two directions, and moreover, that this D3-brane solution is self-dual under S-duality, we conclude that these two theories must be the same.

For $a u \ll 1$, as long as $a u \gg g_{s}^{\prime}$ we can still trust the NS5-branes solution. In this regime, setting $a u=e^{\Phi / \sqrt{N b^{\prime}}}$ we have:

$$
d s^{\prime 2}=\frac{l_{s}^{\prime 2}}{b^{\prime}}\left[d x_{\|}^{2}+d \Phi^{2}+N b^{\prime} d \Omega_{3}^{2}\right], \quad g_{s}(\Phi)=g_{s}^{\prime} e^{-Q \Phi}
$$

where $Q=\frac{1}{\sqrt{N b^{\prime}}}$.

Finally, for the case where $a u \ll g_{s}^{\prime}$, we have to use the S-dual picture, which maps the theory to an ordinary commutative D5-branes.

Using the same variable as above, solution (7) can be written as follows:

$$
\begin{aligned}
& \frac{b}{l^{2}} d s^{2}=k^{-1 / 2}\left(d x_{4}^{2}+d x_{5}^{2}\right)+k^{1 / 2}\left(d x_{\|}^{2}+d \Phi^{2}+N b^{\prime} d \Omega_{3}^{2}\right) \\
& g^{2}(\Phi)=g_{s}^{\prime 2} e^{-2 Q \Phi} k, \quad k=1+e^{2 Q \Phi} .
\end{aligned}
$$

From this form of the solution, one can see the deformation of the linear dilaton background manifestly. The differential equation for the scalar in this background, setting $\Psi=\psi e^{i \omega t}$, is:

$$
\partial_{\Phi}^{2} \psi+2 Q \partial_{\Phi} \psi+\omega^{2} \psi=0 .
$$

This equation has a wave-like solution if $\omega>Q$. Actually one can use this equation to study the absorption cross section of the polarized graviton in this background. Doing so, we find 22] that the absorption cross section can be non-zero at the decoupling limit for an energy

$$
\omega>\frac{b^{\prime-1 / 2}}{\sqrt{N}} .
$$

We note that this is very similar to what was found in [18 for Type IIA NS5branes. This relation, together with what we have had till now, could suggest that $b^{\prime}$ (or $b$ ) has a role of scale in non-commutative NS5-branes theory. From the smeared D3-branes point of view, this can be considered as a scale that measures the smeared directions 
We can also calculate the Wilson loop (or 't Hooft loop) for the smeared D3branes that appeared above. Doing so we will find the same problem as ordinary D5-branes [23] namely that the distance $L$ between quark and antiquark does not depend on $u_{0}$ (where $u_{0}$ is the minimal value of $u$ ). In fact we have

$$
L \sim \sqrt{b^{\prime}} \sqrt{N}
$$

Since this system is equivalent to a non-local theory, this classical minimal distance should be related to the scale of non-locality and in fact this is the case, except for the fact that it is larger by a factor of $\sqrt{N}$ than the naively expected scale which was already mentioned in [8, 7].

Following [24] we can also study the T-duality of the theory on the D5-branes in the presence of a B field as well as non-commutative NS5-branes, which has to be related to the Morita equivalent of the non-commutative theories on the compact space.

\section{Non-commutative Type IIA NS5-branes}

\section{Decoupling limit}

The decoupling limit of the Type IIA NS5-branes can be obtained by T-duality from the Type IIB one. Unlike the ordinary case, where the decoupling limit for both IIB and IIA was the same, here we actually find that these limits are different. The decoupling limit of the Type IIA NS5-brane in the presence of a non-zero RR field along its worldvolume is defined as follows:

$$
l_{s} \rightarrow 0, \quad l_{s}^{3} A=\text { fixed }, \quad g_{s} l_{s}^{-1}=\text { fixed }
$$

where $A$ is the RR field. As we see, this decoupling limit is completely different from the one we have in the ordinary NS5-branes and in fact it is exactly the decoupling limit of smeared ordinary D2-branes. We will go back to this point later. The power of 3 in the fixed quantity $l_{s}^{3} A$ can be understood by the fact that in Type IIA we are dealing with an RR 3-form. We also note that this decoupling limit is different from the one considered in [19], where the same theory has been considered in the DLCQ context; it is not clear to me whether these two definitions are related or if they lead to two different deformations of the NS5-branes theory. ${ }^{5}$

\section{Supergravity description}

The supergravity solution of the Type IIA NS5-branes in the presence of a nonzero RR field is as follows:

$$
d s=h^{-1 / 2}\left[-d x_{0}^{2}+d x_{1,2}^{2}+h d x_{3,4,5}^{2}+f\left(d r^{2}+r^{2} d \Omega_{3}^{2}\right)\right]
$$

\footnotetext{
${ }^{5} \mathrm{I}$ would like to thank O. Aharony for a discussion on this point.
} 


$$
\begin{array}{rlrl}
f & =1+\frac{N l_{s}^{2}}{\cos \theta r^{2}}, & h^{-1}=\sin ^{2} \theta f^{-1}+\cos ^{2} \theta, \\
e^{2 \phi} & =g_{s}^{2} f h^{-1 / 2}, & A_{345}=\frac{\tan \theta}{g_{s}} f^{-1} h \\
A_{012} & =\frac{\sin \theta}{g_{s}} f^{-1} . & &
\end{array}
$$

The decoupling limit is defined by taking the limit $l_{s} \rightarrow 0$ and keeping fixed

$$
\begin{array}{ll}
u=\frac{r}{l_{s}^{2}}, & \bar{g}_{s}=g_{s} l_{s}^{-1}, \\
b=l_{s}^{2} \tan \theta, & \bar{x}_{3,4,5}=\frac{b}{l_{s}^{2}} x_{3,4,5},
\end{array}
$$

From the NS5-branes point of view, we would expect, in the decoupling limit, the mass of a D2-brane stretched between two NS5-branes to be fixed. If it had been the case, we would have had $\frac{r}{g_{s} l_{s}^{3}}$ fixed. But here, in the decoupling limit, we have $\frac{r}{l_{s}^{2}}$ fixed, which means that we are dealing with wrapped NS5-branes. On the other hand, this is the only decoupling limit of NS5-branes that is consistent with string theory dualities. Therefore what we are really studying in this section is noncommutative NS5-branes theory wrapped on a circle. This can also be understood from the T-duality we used to find the decoupling limit. As a result, beside the fixed quantities defined in (17), we also have $\frac{R_{s}}{g_{s} l_{s}}$ fixed (here $R_{s}$ is the radius of the compacted direction). This extra condition will play an interesting role in the phase diagram of the theory. Moreover, there is another parameter in the theory, which we have to take into account: $\beta=g_{s}^{2} b$ [10].

In the decoupling limit the supergravity solution reads:

$$
\begin{aligned}
d s & =\frac{l_{s}^{2}}{b} h^{1 / 2}\left[-d x_{0}^{2}+d x_{1,2}^{2}+h^{-1} d x_{3,4,5}^{2}+\frac{N b}{u^{2}}\left(d u^{2}+u^{2} d \Omega_{3}^{2}\right)\right] \\
h & =1+(a u)^{2}, \quad a^{2}=\frac{b}{N}, \quad e^{2 \phi}=\bar{g}_{s}^{2} b \frac{\sqrt{1+(a u)^{2}}}{(a u)^{2}} \\
A_{012} & =\frac{l_{s}^{3}}{\bar{g}_{s} b^{2}}(a u)^{2}, \quad A_{345}=\frac{l_{s}^{3}}{\bar{g}_{s} b^{2}} \frac{(a u)^{2}}{1+(a u)^{2}} .
\end{aligned}
$$

An important point for this theory is that, in the limit where non-commutative effects are not important and where, moreover, we have to lift the theory to Mtheory, the extra condition mentioned above means $\frac{R_{s}}{R_{11}}$ fixed, with $R_{11}$ the radius of the 11th direction. This means that these circles have to be in the same order or, in other words, that these two circles will decompactify at the same energy. Therefore in the extreme IR we will end up with non-compact $(0,2)$ theory. On the other hand, in the extreme UV limit where the non-commutative effects are important, au $\gg 1$, the solution reduces to:

$$
l_{s}^{-2} d s=\frac{u}{R^{2}}\left(-d x_{0}^{2}+d x_{1,2}^{2}\right)+\frac{R^{2}}{u}\left(d u^{2}+u^{2} d \Omega_{3}^{2}\right)+\frac{R^{2}}{b^{2} u} d x_{3,4,5}^{2}
$$




$$
R^{2}=\sqrt{N b}, \quad e^{2 \phi}=\bar{g}_{s}^{2} \frac{R^{2}}{u}
$$

Moreover, we have a non-zero 3 -form along the directions $(0,1,2)$ and $(3,4,5)$, the latter of which can be gauged away. Therefore we end up with an ordinary D2-branes solution smeared in three directions 5 . Actually we could reach the same conclusion from Type IIB NS5-branes as from using T-duality as we did.

As the energy starts increasing from the IR limit, there are two possibilities. If $\beta \gg 1$, first we reach a regime where we have the wrapped M5-branes in the presence of a $\mathrm{C}$ field here the effects of the $\mathrm{C}$ field are important and we then have to go to the Type IIA theory where the theory is described by smeared D2-branes. On the other hand, if $\beta \ll 1$, we have to go to the Type IIA description, where the noncommutative effects are not important and we can trust the supergravity solution of NS5-branes; in fact, setting $a u=e^{\Phi / \sqrt{N b}}$, we reach the same linear dilaton regime as in the Type IIB case

$$
d s^{2}=\frac{l_{s}^{2}}{b}\left[d x_{\|}^{2}+d \Phi^{2}+N b d \Omega_{3}^{2}\right], \quad g_{s}^{2}(\Phi)=\bar{g}_{s}^{2} b e^{-2 Q \Phi},
$$

and finally we will reach at UV the regime that is described by smeared D2-branes.

As in the Type IIB case we can write the solution in terms of the new variable defined above

$$
\begin{aligned}
\frac{b}{l^{2}} d s^{2} & =k^{-1 / 2}\left(d x_{3}^{2}+d x_{4}^{2}+d x_{5}^{2}\right)+k^{1 / 2}\left(d x_{\|}^{2}+d \Phi^{2}+N b^{\prime} d \Omega_{3}^{2}\right) \\
g^{2}(\Phi) & =\bar{g}_{s}^{\prime 2} b e^{-2 Q \Phi} k^{1 / 2}, \quad k=1+e^{2 Q \Phi}
\end{aligned}
$$

which shows the deformation of linear dilaton background in the presence of an RR field.

One can also study this system using M5-branes in the presence on a non-zero $\mathrm{C}$ field. Using the solution of M5-branes in the presence of a non-zero C field [8, 10], in the near-horizon region we have

$$
\begin{aligned}
d s^{2} & =(N A)^{2 / 3}\left(1+\eta A^{-1}\right)^{1 / 3} l_{p}^{2}\left[A^{-1}\left(d y_{0,1,2}^{2}+\frac{d y_{3,4,5}^{2}}{1+\eta A^{-1}}\right)+d \chi^{2}+d \rho^{2}+\rho^{2} d \Omega_{3}^{2}\right] \\
A & =\sum_{n=-\infty}^{n=\infty} \frac{\pi}{\left[\rho^{2}+(\chi-2 \pi n)^{2}\right]^{3 / 2}}, \quad \rho=\frac{r}{R_{11}}, \quad \chi=\frac{x_{11}}{R_{11}}
\end{aligned}
$$

moreover, we rescaled the coordinates along the branes by $y_{0,1,2}=\sqrt{\frac{\cos \theta}{l_{s}^{2} N}} x_{0,1,2}$ and $y_{3,4,5}=\sqrt{\frac{1}{l_{s}^{2} N \cos \theta}} x_{3,4,5}$. The non-commutative NS5-branes solution is the regime

\footnotetext{
${ }^{6}$ The curvature of the metric is $l_{s}^{2} \mathcal{R} \sim \frac{1}{R^{2} u}$; therefore one can trust the solution at UV. We note that this is in contrast with what we have for non-smeared D2-branes where, at UV, we have perturbative SYM theory.
} 
where $\rho \gg 1$, while $\rho \ll 1$ is the M5-branes geometry. We note that $\eta=\frac{R_{11}^{2}}{l_{s}^{2} N \cos \theta}$ is equal to $\frac{\beta}{N}$ at the decoupling limit; as we can see from the solution for $\eta \ll 1$ we will reach the linear dilaton regime before we go to the non-commutative NS5-branes regime, but for $\eta \gg 1$ we will reach the non-commutative NS5-branes regime when we go to the Type IIA description.

This solution can be used in order to study the absorption cross section of the polarized graviton along the branes. Using the same notation as in [18, the equation for minimally scalar of the form $\Psi=\psi e^{-i \sqrt{s} y_{0}}$ reads

$$
\partial_{\chi}^{2} \psi+\frac{1}{\rho^{3}} \partial_{\rho} \rho^{3} \partial_{\rho} \psi+\sum_{n=-\infty}^{n=\infty} \frac{s \pi}{\left[\rho^{2}+(\chi-2 \pi n)^{2}\right]^{3 / 2}} \psi=0
$$

where $s=\frac{\omega^{2} N l_{s}^{2}}{\cos \theta}$. From this notation one can use the results of [18; in particular we can compute the two-point functions of the theory for the operator that couples to the graviton, which can be interpreted as a component of the energy-momentum tensor of the theory. In fact the results are the same but of course with our $s$ defined above. We also note that as the ordinary NS5-branes, the absorption cross section can be non-zero at the decoupling limit for the case $s>1$, or for the energy larger than $\omega>\frac{b^{-1 / 2}}{\sqrt{N}}$.

This result is the same as for the ordinary case except, that $b$ plays the role of the scale of the theory as expected. From this scale we would expect the mass gap that could appear at $s=1$, as the ordinary NS5-branes, to be of the order of this scale; however it is smaller by a factor of $\sqrt{N}$. Actually this is also the case in the ordinary NS5-branes; while the scale of the theory is $m_{s}$, the mass gap is of the order of $\frac{m_{s}}{\sqrt{N}}$. We also note that, as we saw in the Type IIB case, there is a factor $\sqrt{N}$ difference between the scale of non-locality, which we see from gravity calculation, and what is expected from world-sheet calculation.

We would also like to note that the same phenomenon has been observed for the Coulomb branch of the $\mathcal{N}=4$ SYM theory in 4 dimensions, where the mass gap is

smaller than expected, from a gauge theory point of view, by a factor of $\sqrt{g_{Y M}^{2} N}$ 27]. This fact, together with our previous observation, might mean that there are some stringy effects that change the behaviour of the theory at strong coupling.

\section{Discussion}

We could consider NS5-branes in the presence of a non-zero RR field with higher rank. In this case we would find non-commutative NS5-branes in their decoupling limit. These solutions have been given in [10]. Here we were only interested in the case where, at UV, we can trust the NS5-branes solution; in other words we are interested in the case where, at UV, the theory is thought to be a non-commutative version of the little string theory. In fact this is only the case when we have the smallest rank for the RR field. This can be seen for example from the phase diagram 
of D5-branes with a higher-rank B field [10]. So in this paper we only considered the solution with smallest rank.

Another point that we observed is that the non-commutative little string theories can be described by ordinary branes smeared in some directions. For example the non-commutative Type IIB NS5-branes can be described by the ordinary D3-branes smeared in two directions. If this is really the case, it means that the theory must be equivalent to a non-commutative SYM theory with 16 supercharges in 6 dimensions. On the other hand we know that ordinary SYM theory in 6 dimensions is not renormalizable; in other words, in order to define the theory we need some more information at UV. A way to solve this problem is to assume that the theory flows to a fixed point at UV where we have the little string theory. Now, from our discussion we see that there is another way to make the theory to be well-defined. In this way the theory flows to a non-commutative theory, which can be considered as either non-commutative 6-dimensional SYM theory or non-commutative NS5-branes theory. So non-commutativity gives us another way to make the theory well-defined. This is also the case for the 5-dimensional theory, where there were two possibilities: either it flows to a fixed point at UV $((0,2)$ theory) or it has a non-commutative 5-dimensional description at UV.

We found that the decoupling limit of the Type IIA NS5-branes can be defined only when the theory is wrapped on a circle. This is also consistent with our knowledge of a possible non-commutative version of NS5-branes, since this theory is only defined in the DLCQ context, where one direction is automatically wrapped on a circle [3, 19]. Nevertheless, the theory flows to the ordinary non-compact $(0,2)$ theory at the IR limit.

We also note that the relation between non-commutative D5-branes, NS5-branes and D3-branes smeared in two directions might give us some information about the Coulomb branch of the $\mathcal{N}=4$ SYM theory in four dimensions [25, 26, 27, 28]. 1]

\section{Acknowledgements}

I would like to thank O. Aharony, A. Brandhuber, A. Giveon, A. Kumar and G. Mandal for useful discussions. I am also indebted to Y. Oz for interesting discussions, which motivated me to study this subject, as well as for his comments. The work was partially supported by H. Hofer.

\section{References}

[1] M.R. Douglas, C. Hull, "D-branes and the non-commutative Torus", JHEP 9802 (1998) 008; hep-th/9711165.

[2] M.M. Sheikh-Jabbari, "Super Yang-Mills Theory on Noncommutative Torus from Open Strings Interactions", Phys.Lett. B450 (1999) 119; hep-th/9810179.

\footnotetext{
${ }^{7}$ This issue has been suggested by A. Giveon
} 
[3] N. Seiberg, E. Witten, "String Theory and non-commutative Geometry", JHEP 9909 (1999) 032; th/9908142.

[4] J. Ellis, P. Kanti, N.E. Mavromatos, D.V. Nanopoulos, E. Winstanley, "Decoherent Scattering of Light Particles in a D-Brane Background", Mod.Phys.Lett. A13 (1998) 303; hep-th/9711163.

[5] J.M. Maldacena, "The Large N Limit of Superconformal Field Theories and Supergravity", Adv.Theor.Math.Phys. 2 (1998) 231; Int.J.Theor.Phys. 38 (1999) 1113; hep-th/9711200.

[6] O. Aharony, S.S. Gubser, J. Maldacena, H. Ooguri, Y. Oz, "Large N Field Theories, String Theory and Gravity", hep-th/9905111.

[7] A. Hashimoto, N. Itzhaki, "Non-Commutative Yang-Mills and the AdS/CFT Correspondence", Phys.Lett. B465 (1999) 142; hep-th/9907166.

[8] J.M. Maldacena, J.G. Russo, "Large N Limit of Non-Commutative Gauge Theories", JHEP 9909 (1999) 025; hep-th/9908134.

[9] M. Li, Y.S. Wu, "Holography and non-commutative Yang-Mills", hepth/9909085.

[10] M. Alishahiha, Y. Oz, M.M. Sheikh-Jabbari, "Supergravity and Large N noncommutative Field Theories", JHEP 9911 (1999) 007; hep-th/9909215.

[11] J.L.F. Barbon, E. Rabinovici, "On 1/N Corrections to the Entropy of noncommutative Yang-Mills Theories", JHEP 9912 (1999) 017; hep-th/9910019.

[12] T. Harmark, N.A. Obers, "Phase Structure of Non-Commutative Field Theories and Spinning Brane Bound States", hep-th/9911169.

[13] N. Seiberg, "Matrix Description of M-theory on $T^{5}$ and $T^{5} / Z_{2}$ ", Phys.Lett. B408 (1997) 98:hep-th/9705221.

[14] M. Berkooz, M. Rozali, N. Seiberg, "Matrix Description of M-theory on $T^{4}$ and T" ", Phys.Lett. B408 (1997) 105; hep-th/9704089.

[15] R. Dijkgraaf, E. Verlinde, H. Verlinde, "BPS Quantization of the Five-Brane", Nucl.Phys. B486 (1997) 89; hep-th/9604055.

R. Dijkgraaf, E. Verlinde, H. Verlinde, "5D Black Holes and Matrix Strings", Nucl.Phys. B506 (1997) 121; hep-th/9704018.

[16] O. Aharony, "A brief review of little string theories", hep-th/9911147.

[17] O. Aharony, M. Berkooz, D. Kutasov, N. Seiberg, "Linear Dilatons, NS5-branes and Holography", JHEP 9810 (1998) 004; hep-th/9808149. 
[18] S. Minwalla, N. Seiberg, "Comments on the IIA NS5-brane" JHEP 9906 (1999) 007 hep-th/9904142.

[19] O. Aharony, M. Berkooz, "IR Dynamics of $\mathrm{d}=2, \mathrm{~N}=(4,4)$ Gauge Theories and DLCQ of Little String Theories", JHEP 9910 (1999) 030; hep-th/9909101.

[20] J.X. Lu, S. Roy, “( $p-1)$-Dimensional non-commutative Yang-Mills and $\mathrm{D}_{(p-2)}$ Branes", hep-th/9912165.

[21] R.G. Cai, N. Ohta, "non-commutative and ordinary Super Yang-Mills on $\left(\mathrm{D}_{(p-2)}, \mathrm{D}_{p}\right)$ Bound States", hep-th/0001213.

[22] M. Alishahiha, H. Ita, Y. Oz, to appear.

[23] A. Brandhuber, N. Itzhaki, J. Sonnenschein, S. Yankielowicz, "Wilson Loops, Confinement, and Phase Transitions in Large N Gauge Theories from Supergravity", JHEP 9806 (1998) 001; hep-th/9803263.

[24] A. Hashimoto, N. Itzhak, "On the Hierarchy Between Non-Commutative and Ordinary Supersymmetric Yang-Mills", JHEP 9912 (1999) 007; hepth/9911057.

[25] P. Kraus, F. Larsen, S.P. Trivedi, "The Coulomb Branch of Gauge Theory from Rotating Branes", JHEP 9903 (1999) 003; hep-th/9811120.

[26] D.Z. Freedman, S.S. Gubser, K. Pilch, N.P. Warner, "Continuous distributions of D3-branes and gauged supergravity", hep-th/9906194.

[27] A. Brandhuber, K. Sfetsos, "Wilson loops from multicentre and rotating branes, mass gaps and phase structure in gauge theories", hep-th/9906201.

[28] I. Chepelev, R. Roiban, "A note on correlation functions in $A d S_{5} / S Y M_{4}$ correspondence on the Coulomb branch", Phys.Lett. B462 (1999) 74; hepth/9906224. 